\title{
Pemphigus herpetiformis : A rare clinical variant of pemphigus
}

\author{
Shrestha $P^{1}$, Tajhya $R B^{2}$, Pokharel $A^{3}$ \\ ${ }^{1,2}$ Consultant Dermatologist, Department of Dermatology, Vayodha Hospital Pvt. Ltd, Balkhu, \\ Kathmandu, Nepal \\ ${ }^{3}$ Consultant Dermatologist \& Dermatopathologist, Department of Dermatology, Chitwan Medical \\ College, Bharatpur, Nepal
}

\begin{abstract}
Pemphigus herpetiformis is an autoimmune blistering disease. We report a case of pemphigus herpetiformis, a generalized form, manifested as pruritic grouped vesicles at extremities and trunk, in a seventy years old man. Neutrophil dominant inflammatory substrate was seen in histopathology. Direct immunofluorescene revealed $\operatorname{IgG}$ reactivity in net like pattern at upper epidermis, confirming pemphigus. The patient responded well to prednisolone and colchicine combination initially and maintained remission later on colchicine alone. Colchicine has anti-mitotic, anti-inflammatory and immunosuppressive mechanism of actions and proven benefits in neutrophilic dermatoses. Therefore we recommend colchicine as mono therapy or in combination with immunosuppressive, for the treatment of pemphigus herpetiformis where neutrophil is predominant inflammatory infiltrate histologically.
\end{abstract}

Keywords: Pemphigus, Herpetiform, Pruritic, Vesicles

\section{Address for correspondence}

Dr. Prativa Shrestha

Department of Dermatology

Vayodha Hospitals Pvt Ltd, Balkhu Nepal

Email: shrestha_prativa@yahoo.com

\section{Introduction}

Pemphigus is an autoimmune blistering disease. Many variants have been described but pemphigus herpetiformis is relatively uncommon. Clinically it presents as dermatitis herpetiformis and immunopathologically as pemphigus. Incidence is equal in both genders, with mean age of onset around 60 years. These patients usually present with intensely pruritic vesicles and blisters with variable duration. Two forms have been described; generalized and localized types. There is a paucity of studies on pemphigus herpetiformis, so whether it is an atypical variant of pemphigus foliaceus or it is separate entity is so far not known.

\section{Case Report}

The patient, a 70-year-old man consulted for recurrent episodes of an intensely pruritic skin lesions over trunk and limbs persisting for six months duration. Initially it appeared on limbs predominantly extensor aspects and approximately a month later it progressed to involve sacral region and upper chest. He was prescribed multiple courses of oral and topical antibiotics until he visited to us. On reviewing his past medical history, he has hypertension and had undergone post percutaneous transluminal coronary angioplasty six years ago and since then he was taking cardiac medicines namely asprin $75 \mathrm{mg}$, 


\section{Case Report}

atorvastatin $10 \mathrm{mg}$, amlodipine $5 \mathrm{mg}$ and dilitazem $60 \mathrm{mg}$. He had unexplained iron deficiency anaemia (hemoglobin- $8.4 \mathrm{gm} / \mathrm{dl}$, ferritin$4.73 \mathrm{ng} / \mathrm{ml}$, total iron binding capacity-386ug/dl, transferring saturation $\%-10 \%$ ), vitamin B12 deficiency $(155.4 \mathrm{pg} / \mathrm{nl})$ and blunting of duodenal villi with presence of helicobacter pylori in the endoscopic biopsy. Occult blood test was negative. After taking iron and vitamin B12 supplements, his hemoglobin increased to $11 \mathrm{gm} / \mathrm{dl}$. Apart from occasional epigastric pain, he does not give history of any gastrointestinal and neurological symptoms. He doesn't recall worsening of the skin conditions with any particular diet. Clinically his iron deficiency anemia and blunting of duodenal villi couldn't be correlated with his skin condition.

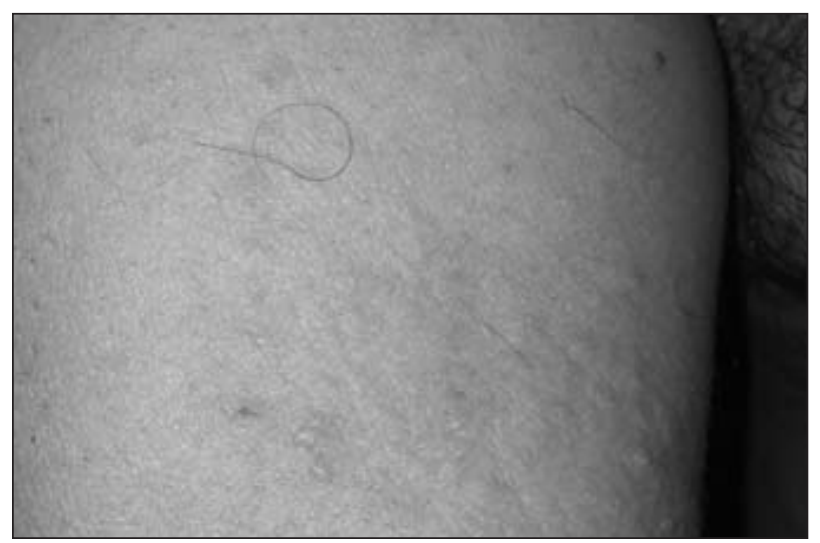

Figure 1: Intensely pruritic vesicles and urticated papules arising on an erythematous base at right arm

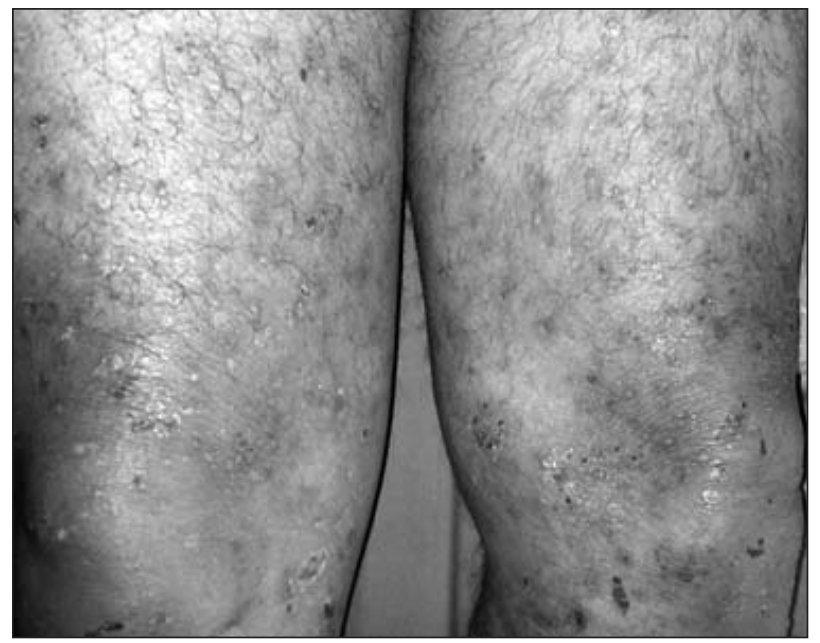

Figure 2: Excoriated scabbed lesions resolving with post inflammatory hypopigmentation symmetrically at bilateral lower limbs

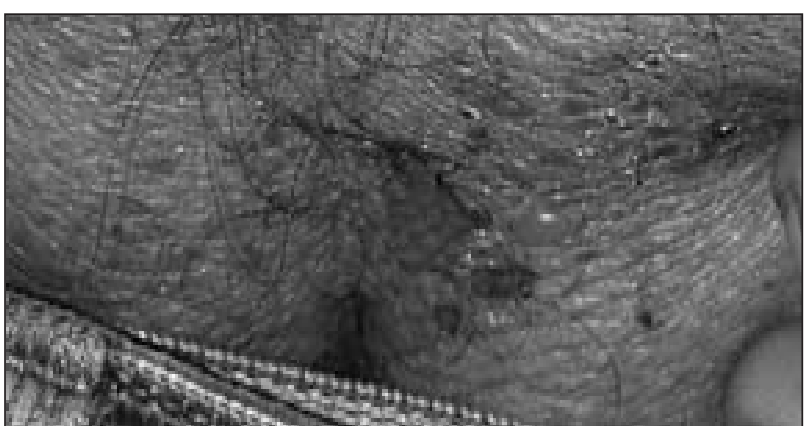

Figure 3: Few intact grouped vesicles with superficial de-epithelization at the sacral region

On physical examination, he had few intact itchy grouped vesicles arising on normal to urticated skin and crusted scabbed lesions over the upper chest, sacral region, extensor of both upper and lower limbs bilaterally (Figure 1, 2 \& 3). These lesions healed with faint hypopigmentation and no scarring. Nikolsky's sign was negative. Clinically no secondary infection was seen. Mucous membrane, nail, scalp, palms and soles were spared. Rest of the physical examination was normal. So a presumptive diagnosis of dermatitis herpetiformis with differential diagnoses of bullous pemphigoid- vescicular type, pemphigus foliaceus- dermatitis herpetiformis and bullous lupus was made.

Skin biopsy was taken from left arm. Histologically epidermis showed mild acanthosis, collection of neutrophils at malphigian layer of the epidermis with few areas of microabcess (Figure 4). Upper dermis showed mild papillary dermal edema with few collection of neutrophils. No clear separation or cleft was seen. Hence direct immunofluorescence (DIF) was suggested for confirmation.

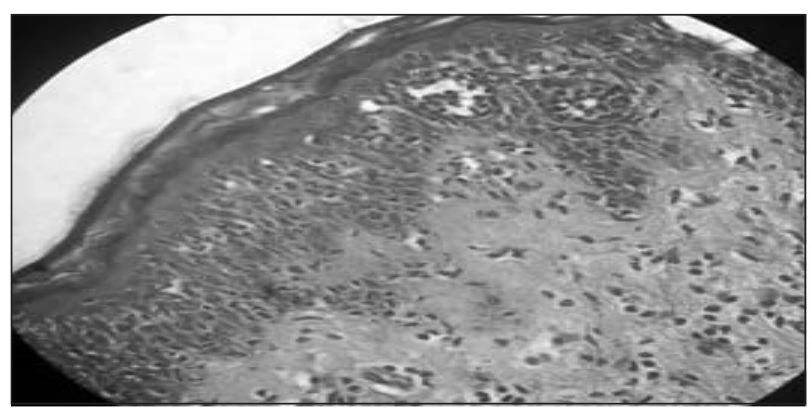

Figure 4: Histopathology showing neutrophilic spongiosis with papillary dermal edema 


\section{Case Report}

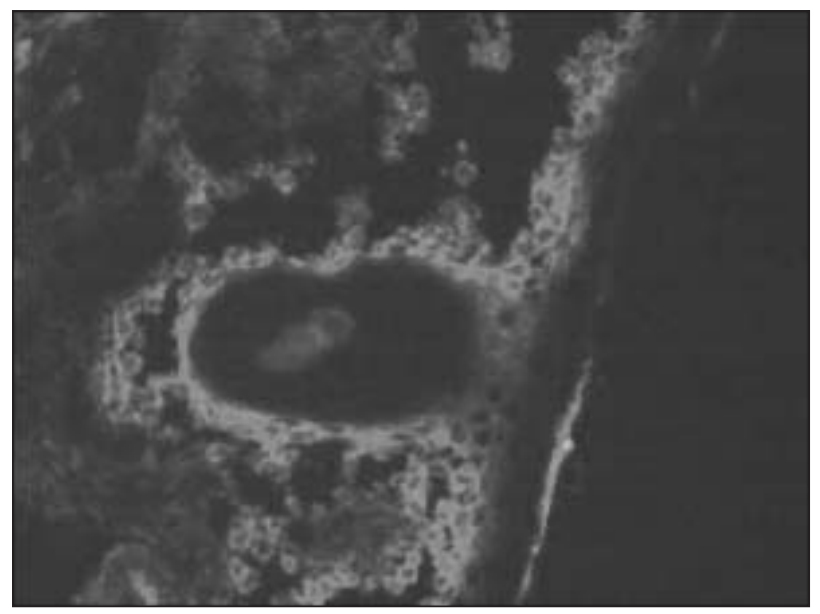

Figure 5: Direct immunofluorescence showing IgG reactivity in net like pattern in upper epidermis DIF performed on perilesional normal looking skin showed deposits of IgG in net like pattern in the upper epidermis compatible with pemphigus (Figure 5). Immunofluorescence for IgA, IgM, $\mathrm{C} 3$ and $\mathrm{C} 1 \mathrm{q}$ were negative.

Hence a diagnosis of pemphigus herpetiformis (PHF) was made on the basis of clinical features where dermatitis herpetiformis like intensely itchy grouped vesicles in generalized distribution were present along with immunopathologic features of pemphigus. Treatment was started with prednisone $40 \mathrm{mg}$ /day and topical clobetasol. His itching and occurrence of new lesion substantially reduced, so after two weeks steroid was tapered to $20 \mathrm{mg}$ /day and further reduced and colchicine $0.5 \mathrm{mg}$ twice daily was added for next two weeks. No recurrence was noted at subsequent follow-up, therefore systemic steroid was finally stopped and was continued only on colchicine for next two months only. There was no relapse at six months after all the drugs were stopped. He continued his previous cardiac medicines and vitamin supplements during the treatment and remission.

\section{Discussion}

Pemphigus herpetiformis (PHF) was first introduced by Jablonska and colleagues as a variant of pemphigus. ${ }^{1}$ PHF is atypical variant of pemphigus that combines the clinical features of dermatitis herpetiformis with the immunopathologic features of pemphigus. Mean age of onset around is 60 years. ${ }^{2}$ However childhood cases has also been reported. ${ }^{3}$ Patients affected with pemphigus herpetiformis usually have a subacute onset of disease. Approximately half the patients experience severe pruritus. The clinical distribution of PHF is almost invariably generalized, involving both the trunk and limbs, characteristic skin manifestations are pruritic erythematous vesicular, bullous or papular lesions in herpetiform pattern, similar to those of dermatitis herpetiformis. ${ }^{4}$ However localized form of PHF has been described. ${ }^{5}$ PHF occurrence in association with malignancies such as lung cancer, ${ }^{6,7}$ prostate cancer, ${ }^{8}$ and cutaneous angiosarcoma ${ }^{9}$ has also been reported. Histologically demonstrated eosinophil and/or neutrophil infiltration into the epidermis may be relevant pathogenically in the disease process of pemphigus herpetiformis. Huhn et al found that the inflammatory infiltrate in patients with PHF was $68 \%$ eosinophil-dominant, $16 \%$ neutrophildominant, and $16 \%$ mixed eosinophil/neutrophil. ${ }^{10}$ In the neutrophil-dominant subset, epidermal cells secrete a neutrophil chemokine interleukin 8 (IL8 ), which apparently is induced by $\mathrm{IgG}$ autoantibodies to desmoglein and may be responsible for the recruitment of neutrophils to the epidermis, resulting in the subsequent blistering process. ${ }^{2}$ Pemphigus herpetiformis appears to be mediated by the immunoglobulin G (IgG) class of autoantibodies that target the skin epidermis desmoglein components, commonly Desmoglein $1 .^{2}$

DIF helps detect molecules such as immunoglobulins and complement components within biopsy specimens. ${ }^{11}$ For bullous diseases, DIF is performed using perilesional skin, that is, normal-appearing skin immediately adjacent to a lesion (vesicle, bulla, urticarial plaque, or erythematous patch). ${ }^{12}$ Indirect immunofluorescence (IIF) confirms the presence in the patient's serum of IgG-circulating autoantibodies that bind to epidermal cell surfaces. The titer of autoantibodies often parallels the clinical level of activity of pemphigus herpetiformis and often is helpful in following the patient's disease activity after the diagnosis has been established. Other tests such as immunoblotting, ELISA, immunoprecipitation, 


\section{Case Report}

chest xray, prostate specific antigen are recommended if symptoms suggest.

Pemphigus herpetiformis is responsive to antiinflammatory and immunosuppressive medications. Dapsone is the drug of choice if the patient tolerates the treatment, systemic corticosteroid and immunosuppressives, monoclonal antibody anti-CD20 (rituximab). ${ }^{13}$ We treated our patient with prednisolone and colchicine combination initially and colchicine alone later as a steroid sparing agent. Colchicine has anti-mitotic, anti-inflammatory and immunosuppressive mechanism of actions and proven benefits in neutrophilic dermatoses. ${ }^{14}$ In our patient, neutrophil dominant infiltrate was seen histologically and he maintained remission on colchicine alone and 6 months after it was stopped.

\section{Conclusion}

We would like to stress the importance of performing direct immunofluorescence to test for the presence of an autoimmune blistering disorder when histology reveals neutrophilic and/or eosinophilic spongiosis for correct diagnosis. Also, colchicine can be safe steroid sparing agent in pemphigus herpetifomis with neutrophil dominant infiltrate.

\section{References}

1. Jablonska S, Chorzelski TP, Beutner EH, Chorzelska J. Herpetiform pemphigus, a variable pattern of pemphigus. Int J Dermatol 1975; 14: 353-9. http://dx.doi.org/10.1111/j.13654362.1975.tb00125.x

2. Sandhu N. Pemphigus Herpetiformis. Available from: http://emedicine.medscape.com/article/1064550-overview. Accessed on 15th January 2015.

3. Duarte IB, Bastazini I Jr, Barreto JA, Carvalho CV, Nunes AJ. Pemphigus herpetiformis in childhood. Pediatr Dermatol 2010; 27: 488-91. http://dx.doi.org/10.1111/j.1525-1470.2010.01256.x

4. Robinson ND, Hashimoto T, Amagai M, Chan LS. The new pemphigus variants. J Am Acad Dermatol 1999; 40: 649-71. http://dx.doi.org/10.1016/S0190-9622(99)70145-3

5. Hung CC, Hung YHW, Kao CH. Localized pemphigus herpetiformis: two case reports. Dermatologica Sinica 2010; 28: 36-9. http://dx.doi.org/10.1016/S1027-8117(10)60006-7

6. Kubota Y, Yoshino Y, Mizoguchi M. A case of herpetiform pemphigus associated with lung cancer. J Dermatol 1994; 21: 609-11.

7. Palleschi GM, Giomi B. Herpetiformis pemphigus and lung carcinoma: a case of paraneoplastic pemphigus. Acta Derm Venereol 2002; 82: 304-5. http://dx.doi.org/10.1080/000155502320323333

8. Marzano AV, Tourlaki A, Cozzani E, Gianotti R, Caputo R. Pemphigus herpetiformis associated with prostate cancer. J Eur Acad Dermatol Venereol 2007; 21: 696-8.

9. Lu Y, Zhang M. Pemphigus herpetiformis in a patient with well-differentiated cutaneous angiosarcoma: case report and review of the published work. J Dermatol 2012; 39: 89-91.

10. Huhn KM, Tron VA, Nguyen N, Trotter MJ. Neutrophilic spongiosis in pemphigus herpetiformis. J Cutan Pathol 1996; 23: 264-9. http://dx.doi.org/10.1111/j.1600-0560.1996.tb01477.x

11. Beutner EH, Kumar V, Krasny SA, Chorzelski TP. Defined immunofluorescence in immunodermatology. In: Beutner EH, Chorzelski TP, Kumar V, editors. Immunopathology of the skin. 3rd ed. John Wiley \& Sons; 1987. p. 3-40. 


\section{Case Report}

12. Mutasim DF, Adams BBD. Immunofluorescence in dermatology. J Am Acad Dermatol 2001; 45: 803-22. http://dx.doi.org/10.1067/mjd.2001.117518

13. Maciejowska E, Jablonska S, and Chorzelski T. "Is pemphigus herpetiformis an entity?" Int J Dermatol 1987; 26: 571-7. http://dx.doi.org/10.1111/j.1365-4362.1987.tb02308.x

14. Konda C, Rao AG. Colchicine in dermatology. Indian J Dermatol Venereol Leprol 2010; 76 : 201-5. http://dx.doi.org/10.4103/0378-6323.60552 